\section{Analysis of pro-arrhythmic effects induced by different routes of administration of bone marrow stem cells}

\author{
Anna Vittoria Mattioli, \\ Roberto Lonardi, ${ }^{2}$ Enrico Giuliani, ${ }^{3}$ \\ Alberto Barbieri, ${ }^{3}$ Sonia Pennella ${ }^{4}$ \\ Alberto Farinetti ${ }^{2}$ \\ 'Department of Biomedical Science; \\ 2Department of Surgery; ${ }^{3}$ Section of \\ Anesthesiology, University of Modena \\ and Reggio Emilia; ${ }^{4}$ Istituto Nazionale di \\ Ricerche Cardiovascolari, UO Modena, \\ Italy
}

\section{Abstract}

There has been repeated concern that intramyocardial (i.m.) delivery of cells could cause ventricular arrhythmias. The aim of the study was to evaluate the pro-arrhythmic effects of bone marrow stem cell (BMSC) injection and compare different routes of administration. An ischemia reperfusion injury was induced in New Zealand rabbits by temporal ligation of anterior descending coronary artery. Homologous BMSCs were isolated, cultured and re-suspended for injection. We compared different routes of BMSC injections, intramyocardial (i.m.) versus intravenous (i.v.) administration of cells. A control group was treated with i.m. injections of saline. The hourly number of supra- and ventricular premature contractions (VPCs), QT interval time and QTc time were recorded and calculated. At Day 7 after cell injections, VPCs were more frequent in the groups treated with i.m. BMSCs and i.m. saline compared with i.v. BMSCs (132 $\pm 19 ; 54 \pm 14$ and $34 \pm 9$, respectively; $\mathrm{P}<0.01$ within groups), whereas at Day 21 the number of VPCs was higher in the 2 groups treated with either i.m. or i.v. BMSCs compared with saline $(96 \pm 23 ; 52 \pm 19$ and $25 \pm 20$, respectively; $\mathrm{P}<0.001$ within groups). QTc time interval was prolonged during ischemia, and recovered in control and in the group treated with i.v. cells, whereas it remained longer in rabbits treated with i.m. BMSCs. These findings show that i.m. BMSC injections induced a high number of pre-arrhythmic events suggesting changes in cardiac electrophysiological properties. The i.v. administration of cells resulted in lower VPC beats and in a temporary QT prolongation. These results suggest that the combination of BMS cells and i.m. injections induced an electrical remodeling that contributed to the development of arrhythmias.

\section{Introduction}

The clinical use of stem cells in acute myocardial infarction has produced conflicting results. ${ }^{1,2}$ Although stem cell-based therapy provides a promising approach to salvage the damaged heart in most animal experiments, and to prevent left ventricular remodeling, outcomes in clinical studies of transplantation of autologous bone marrow stem cells (BMSCs) for cardiogenesis or neovasculogenesis have been inconsistent. ${ }^{3-5}$ Moreover, there have been repeated concerns that delivery of stem cells could cause potentially life-threatening ventricular arrhythmias. ${ }^{6-8}$ Indeed, the major safety issue associated with cardiac cell therapy has been the occurrence of sustained ventricular arrhythmias in some of the patients injected with skeletal myoblasts. ${ }^{7}$ Menasche and co-workers suggested that failure of differentiated myotubes to express gap junction proteins resulted in electrically insulated cell clusters which could slow the conduction velocity of electrical impulses and consequently predispose to re-entry circuits.

An important issue in regenerative therapy in acute myocardial ischemia is that therapeutic efficacy and arrhythmia occurrence induced by cell injection into the post-MI heart may be affected by the cell delivery route. The intramyocardial (i.m.) route can deliver cells selectively into target areas; however, this method could induce mechanical injury and subsequent acute inflammation..$^{910}$ In contrast, the intravenous (i.v.) route can deliver donor cells more homogeneously with less mechanical damage and inflammation in the myocardium, but the rate of homing and engraftment is poor., ${ }^{9}, 10$

Previous clinical studies reported rare incidence of arrhythmias after i.m. injection of BMSCs; however, all patients had been treated with antiarrhythmic drugs and/or beta blockers that could have masked the pro-arrhythmic effect of the treatment. ${ }^{11,12}$ In addition, continuous monitoring may not be sufficient to adequately examine the occurrence of arrhythmia.

The aim of the present study was to investigate the pro-arrhythmic effects of BMSC injection by different routes of cell administration in a pre-clinical model.

\section{Materials and Methods}

\section{Patient selection and study protocol}

In 30 New Zealand rabbits (POLISTAB, UNIMORE Modena, Italy), mean weight $4.1 \pm 0.5$ $\mathrm{kg}$, an ischemia/reperfusion injury was induced by ligation of left anterior descending coronary artery (LAD). The rabbits were sedated by intramuscular (quadriceps femoris)
Correspondence: Anna Vittoria Mattioli, Department of Biomedical Science, University of Modena and R.E. via del Pozzo, 71, 41100 Modena, Italy

Tel: +39.59 .4224043 - Fax: +39.59 .2055426$

E-mail: annavittoria.mattioli@unimore.it

Key words: bone marrow stem cells, intramyocardial injections, ischemia/reperfusion, pre-clinical study, QT interval.

Acknowledgements: part of these data was presented during the Scientific Session of the American College of Cardiology New Orleans, USA, March 2011.

Contributions: AVM, conception and design, obtained funding, data collection, statistical analysis, analysis and interpretation, wrote the article, critical revision, and takes overall responsibility for the manuscript; RL, conception and design, and data collection; AF, conception and design, obtained funding, data collection, analysis and interpretation, critical revision, and overall responsibility for the manuscript; EG, data collection, analysis and interpretation; SP, data collection, analysis and interpretation, and statistical analysis; $\mathrm{AB}$, critical revision of the article. All authors approved the final version of the manuscript for publication.

Conflicts of interests: authors declare no potential conflicts of interests.

Funding: this research was supported by a Grant from the Istituto Nazionale di Ricerche Cardiovascolari, Bologna, Italy.

Received for publication: 14 December 2011 Revision received: 28 February 2012.

Accepted for publication: 29 February 2012

This work is licensed under a Creative Commons Attribution NonCommercial 3.0 License (CC BYNC 3.0).

(C) Copyright A.V. Mattioli et al., 2012

Licensee PAGEPress, Italy

Stem Cell Studies 2012; 2:e1

doi:10.4081/scs.2012.e1

injection of $1-2 \mathrm{mg} / \mathrm{kg}^{-1}$ medetomidyne and 0.7 $1 \mathrm{mg} / \mathrm{kg}^{-1}$ midazolam under the direct supervision of an animal handler in order to reduce stress reaction. The marginal auricular vein was cannulated with a $22 \mathrm{G}$ catheter and saline infusion was started at $1-1.5 \mathrm{~mL} / \mathrm{kg} / \mathrm{min}$. An initial bolus of $2-3 \mathrm{mg} / \mathrm{kg}^{-1}$ of $1 \%$ propofol solution was administered 2 min prior to surgical positioning and every 5 min to maintain anesthesia. A muzzle mask was then positioned over the nose and mouth, and a bag-valve 0.5 1 ventilation system was connected to an oxygen supply. Respiratory rate was monitored by counting reservoir volume variations. A 12 lead electrocardiogram (ECG) was recorded throughout the operation. A left thoracotomy 
was made through the fifth intercostal space and the pericardium was opened. To produce an anterior ischemia, a distal portion of the LAD was selected for ligation. The selected artery was temporarily occluded, and the extent of ischemia was visually and electrocardiographically assessed (ECG ELI 150, Mortara Rangoni Europe srl., Casalecchio di Reno, Bologna, Italy). Ischemia lasted $20 \mathrm{~min}$, then the area was reperfused. A successful induction of ischemia was confirmed by elevation of the ST segment by more than $0.2 \mathrm{mV}$ in leads I, II and aVL. BMSCs were injected by i.v. or by i.m. route. The surgical delivery of the cells was performed with a specific syringe equipped with an angled needle. Animals were treated as follows: Group 1, i.m. injections of BMSCs; Group 2, i.v. injection of BMSCs; and Group 3, i.m. injection of saline (to evaluate pro-arrhythmic effect of i.m. injections). After delivery of the cells, a single chest tube was placed under direct visualization through a 5 $\mathrm{mm}$ site. The chest tube was removed after disconnection from positive pressure ventilation. The edges of the sternum were then brought together and fixed with Ethilon 5.0 sutures. The remaining surgical accesses were closed in two layers. The anesthetic was stopped, the animal was extubated when appropriate and allowed to recover. All animals received postoperative antimicrobial therapy (cephazoline $100 \mathrm{mg} / \mathrm{kg}$ i.m. twice/day for 3 days) and buprenorphine $(0.3 \mathrm{mg}$ i.m. twice/day for 3 days) for post-operative pain.

\section{BM cell collection and culture}

BMSCs were aspirated from posterior iliac crest, were cultured and re-suspended in saline $(\mathrm{NaCl} 0.9 \%)$ for injection at the level of peri-infarcted zone (6 injections). BMSCs were isolated by magnetic separation (MACs Miltenyi Biotec srl., Calderara di Reno, Bologna, Italy).

\section{Bone marrow cell culture}

Cells were cultured in DMED with $10 \%$ fetal bovine serum, penicillin $\mathrm{G}(100 \mathrm{U} / \mathrm{mL})$ and streptomycin $(100 \mathrm{U} / \mathrm{mL})$ at $37^{\circ} \mathrm{C}$ in humid air with $5 \% \mathrm{CO} 2$. After $72 \mathrm{~h}$, the cells that were adherent to the culture flask were maintained for propagation and non-attached cells were discarded by 4 changes of medium. BMSCs were cultured with DMED with $10 \mu \mathrm{mol} / \mathrm{mL}$ BrdU (Abcam, Biochemicals, Cambridge, UK) for $48 \mathrm{~h}$ in order to make the donor cells for further identification. Cultured donor cells were dissociated from the culture dishes with 0.25\% trypsin (Life Technologies Europe BV, Monza, Italy), neutralized with culture medium, and collected by $2000 \mathrm{rpm}$ centrifugation for $10 \mathrm{~min}$ at room temperature according to the manufacturer's instructions. Injected saline included $4 \times 10^{6}$ BMSCs (identified by superficial markers CD34, CD45, CD166) for a total of $0.2 \mathrm{~mL}$. The nuclei of donor cells were labeled with BrdU $72 \mathrm{~h}$ before transplantation; $70 \%$ of nuclei were stained positive for BrdU in culture.

\section{Electrocardiogram data collection and analysis}

An ECG recording system (RTSoft, Novacor, Rueil Malmaison Cedex, France) was localized over the neck of the animals and recordings were made the day before surgery, for $48 \mathrm{~h}$ after surgery, and then for the next seven days. The hourly number of supraventricular (SVPC) and ventricular premature contractions (VPCs), episodes of sustained ventricular tachycardia (VT), ventricular fibrillation (VF), the morphological change over the follow up, and QT time were calculated and analyzed by using specific ECG analysis software.

Electrocardiogram QT time and cycle length (RR interval) were measured manually using calipers. Leads II, aVL, and aVF were recorded before, during, and after the vessel occlusion at an ECG sweep of $25 \mathrm{~mm} / \mathrm{s}$ and amplitude calibration at $10 \mathrm{~mm} / \mathrm{mV}$. Time intervals (QT and RR) were measured in at least five consecutive cardiac cycles and mean values were calculated. QT intervals were measured from the earliest onset of the QRS complex to the end of the T-wave.

Using an intraindividual approach, the same lead was used consistently for all measurements. The point of T-wave offset was defined as the return of the T-wave to baseline. Baseline and occlusion measurements were performed in the same lead. All QT intervals were adjusted for corresponding heart rates. Corrections were performed using the Framingham Formula. ${ }^{13}$

$$
\text { Framingham: } Q T c(F H)=Q T+0.154(1000-R R)
$$

(QT and RR in ms)

The animals were monitored for the next 21 days (time of sacrifice). After sacrifice, the infarcted zone was divided into 6 consecutive parts, numbered 1-6: part 1was in the zone nearest to the upper part of the heart and part 6 in that nearest to the apex. The 6 parts were: right and left ventricles, right and left atria, and interventricular and interatria septa. These parts of the heart allowed us to make a thorough study of cell implantation. cTn I antibody (mouse source IgG1, anti rabbit, Sigma) and BrdUrd antibody (mouse source IgGl, IGN) were made for double marking immunohistochemical examination.

All animals received care in compliance with the European Convention on Animal Care. Animal care was provided by the same trained operator in order to reduce stress. The study was approved by the Research Animal Care and Use Committee of the University of Modena

Table 1. Baseline characteristics of the 3 groups of animals before surgery.

\begin{tabular}{lccc} 
& BMSCs i.m. injection & BMSCs i.v. injection & Saline i.m. \\
Weight $(\mathrm{Kg})$ & $4.1 \pm 0.6$ & $4.0 \pm 0.4$ & $4.1 \pm 0.7$ \\
Female/male & $4 / 4$ & $5 / 3$ & $5 / 3$ \\
\hline Heart rate (b/min) & $200 \pm 16$ & $201 \pm 10$ & $196 \pm 18$ \\
QTC (ms) & $422 \pm 16$ & $419 \pm 20$ & $420 \pm 25$ \\
\hline VPC at Day 0 before surgery & $11 \pm 4$ & $13 \pm 6$ & $14 \pm 5$ \\
SVPC at Day 0 before surgery & $22 \pm 6$ & $19 \pm 7$ & $20 \pm 3$ \\
\hline
\end{tabular}

BMSCs, bone marrow stem cells; QTc, corrected QT interval; VPC, ventricular premature contraction; SVPC, supra-ventricular premature contraction. Data are expressed as mean + SD.

Table 2. Parameters from electrocardiogram analysis.

\begin{tabular}{lccc} 
& BMSCs i.m. injection & BMSCs i.v. injection & Saline i.m. \\
Heart rate at Time 0 (b/min) & $200 \pm 16$ & $201 \pm 10$ & $196 \pm 18$ \\
Heart rate during ischemia (b/min) & $186 \pm 20$ & $199 \pm 25$ & $184 \pm 23$ \\
\hline Heart rate at Day 7 (b/min) & $202 \pm 21$ & $210 \pm 21$ & $208 \pm 14$ \\
Heart rate at Day 21 (b/min) & $200 \pm 24$ & $198 \pm 22$ & $203 \pm 19$ \\
\hline VPC at Time 0 (hourly) & $11 \pm 4$ & $13 \pm 6$ & $14 \pm 5$ \\
VPC at Day 7 (hourly) & $132 \pm 19 * *$ & $34 \pm 9 * *$ & $54 \pm 14^{* *}$ \\
\hline VPC at Day 21 (hourly) & $96 \pm 23^{* *}$ & $52 \pm 19 * *$ & $25 \pm 20$ \\
QTc at Time 0 (ms) & $418 \pm 16$ & $416 \pm 20$ & $415 \pm 25$ \\
\hline QTc during ischemia (ms) & $432 \pm 21^{*}$ & $427 \pm 23^{*}$ & $434 \pm 28^{*}$ \\
QTc at Day 7 (ms) & $429 \pm 19^{*}$ & $421 \pm 19$ & $426 \pm 29$ \\
\hline QTc at Day 21(ms) & $430 \pm 21^{* *}$ & $410 \pm 34$ & $408 \pm 30$ \\
\hline
\end{tabular}

BMSCs, bone marrow stem cells; VPC, ventricular premature contraction; QTc, corrected QT interval; Time 0, day before surgery; ${ }^{*} \mathrm{P}<0.05$ vs Time 0 (pre-ischemia); ${ }^{* *} \mathrm{P}<0.001$ us time 0 (pre-ischemia). 
and Reggio Emilia (Italy) (prot. n. 25, 18/03/2008 and prot. n. 83, 25/09/2009).

\section{Timing of follow up}

ECG was recorded by using a commercial Holter; recording was started from the day before surgery (Time 0 ), continued during the entire surgical procedure (Time 1), and for the seven days following surgery (Time 2). A 24-h ECG-Holter was performed at Day 21 before sacrifice (Time 3 ).

\section{Statistical analysis}

SPSS software, version 14.0.1 (SPSS Inc., Chicago, Ill, USA), was used for statistical analysis. Comparison of data between groups was performed by ANOVA. The t-test was used to compare data obtained by animals of the same group. $\mathrm{P}<0.05$ was considered statistically significant. All data are expressed as mean + SD.

\section{Results}

Of the initial group of 30 rabbits, 6 rabbits developed intractable ventricular fibrillation during the experimental protocol (3 during coronary ligation but before cell injections, 3 following i.m. injections), and these 6 were excluded from the study. Each of these 6 rabbits showed a small pericardial effusion after opening the pericardium; however, no histological changes were found. The remaining 24 rabbits were evaluated for 21 days before sacrifice (mean $22 \pm 2$ days). There were no differences in hemodynamic parameters at baseline between the experimental groups (Table 1).

During the acute phase of ischemia induction, we registered 3 episodes of reversible ventricular tachycardia (VT) and one episode of ventricular fibrillation successfully cardioverted. We also reported 4 episodes of

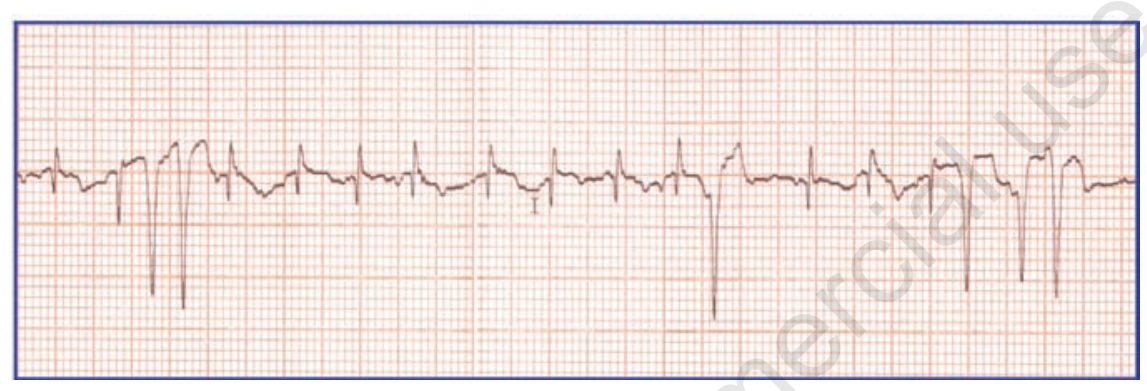

Figure 1. Electrocardiogram recording during ischemia/reperfusion protocol showing several ventricular premature contractions. reversible VT occurring at Day 3 and Day 4 in 2 animals treated with cell injection into the myocardium, whereas no VT was observed in rabbits treated with i.v. injections of cells. Figure 1 shows several VPCs recorded during the ischemia/reperfusion protocol. At Day 7 after surgery, VPCs were more frequent in the groups of animals that had received i.m. injections of BMSCs or saline compared to animals that had received i.v. administration of BMSCs $(132 \pm 19 ; \quad 54 \pm 14$ and $34 \pm 9$, respectively; $\mathrm{P}<0.01$ within groups), whereas at Day 21 the number of VPCs was higher in the group treated with BMSCs compared with saline $(96+23$ BMSCs i.m. vs $52+19$ BMSCs i.v. vs $25+20$ saline i.m.; $\mathrm{P}<0.001$ within groups) (Table 2 ).

Comparison between the 3 groups is shown in Figure 2. During the follow up, we observed an increase in heart rate during meals and in relation to acute stress (i.e. presence of human care). No lethal ventricular events were observed during follow up.

Mean QTc interval for animals during occlusion of the LAD increased in all groups, whereas in the group treated with i.m. injections it remained more prolonged than in the group treated with i.v. injections. After 21 days, QTc was prolonged in the group treated with i.m. injections of BMSCs from $418 \pm 16$ (preischemia) to $429 \pm 19$ (at Day 7) to $430 \pm 21$ (at Day 21) (Figure 3).

Macroscopically, ligation of a distal portion of the LAD resulted in a relatively small, near transmural infarct in the apical part of the left ventricle. At necropsy, histopathology showed absence of chronic inflammatory cell infiltrates or evidence of rejection. However, several inflammatory cells and white foci were pres-

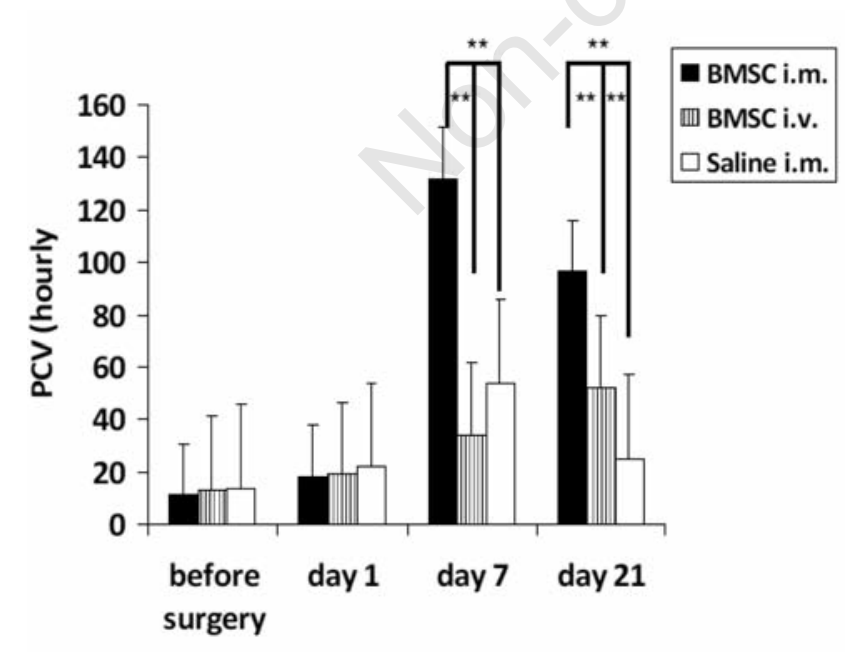

Figure 2. Comparison between the 3 groups: number of ventricular premature contractions in animals treated with i.m. injections of bone marrow stem cells (BMSCs) compared with groups of animals treated with i.v. BMSCs or saline $\left({ }^{* *} \mathrm{P}<0.001\right.$ vs day 0$)$. Full black bars, BMSC i.m.; white bar with vertical black lines bars, BMSC i.v.; full white bars, saline i.m.
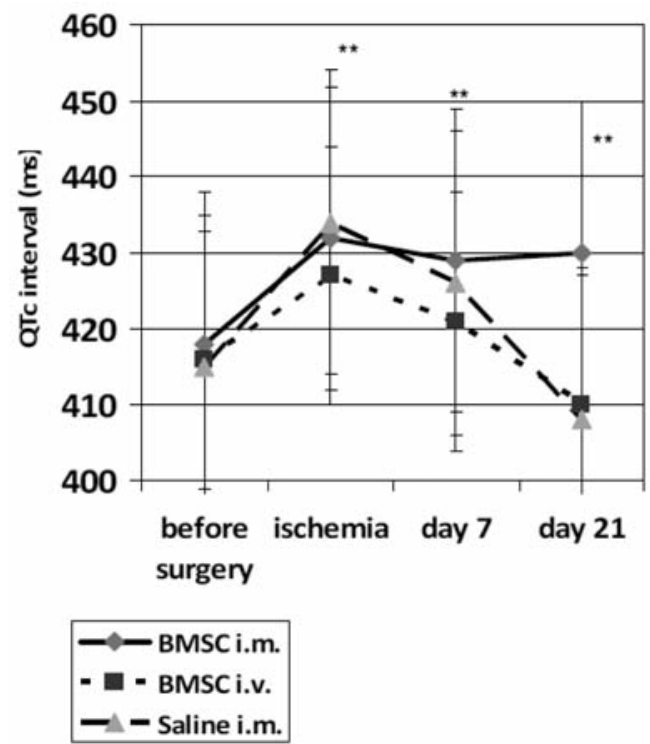

Figure 3. Mean QTc interval in the different groups of treatment over time $\left({ }^{*} \mathrm{P}<0.01\right.$ vs Day 0$)$. 
ent on the endomyocardial surface across all groups. These were higher in the group of animals that received i.m. injections compared to those receiving i.v. injections. During the 21 days following cell transplantation, it was observed that the BrdU-labeled cells at the implanted area were forming cell islets isolated from the native cardiomyocytes. In addition, few BrdU-stained cells were turned to musclelike cells and stained positively for troponin I.

\section{Discussion}

The present study reported on the proarrhythmic effects of BMSC administration in a pre-clinical cardiac ischemia/reperfusion model. The major finding of this study was that the i.m. route of administration induced more ventricular arrhythmic events than systemic administration. Animals receiving BMSCs developed more VPCs and a prolongation of QT time intervals compared with rabbits that received saline injections, suggesting a potential role of cell engraftment in the mechanisms of arrhythmias.

Arrhythmia occurrence induced by cell injection into the injured heart can be affected by the cell delivery route. The i.m. route delivers cells selectively into target areas; however, these cells tend to form islet-like cell clusters that are isolated from the host myocardium. Such local heterogeneity in the myocardium is considered a potential source of arrhythmias. ${ }^{9,10}$ In contrast, the i.v. route of cell administration is affected by a low rate of sustained cell retention due to the cell residue in the syringe and to the cell leakage caused by the myocardial 'squeezing' effect. Subsequently, a great number of the initially retained cells die.

The present study was designed to evaluate the pro-arrhythmic effects of cell injection. During the acute phase of myocardial ischemia, the i.m. administration of cells induced a greater number of VPCs and ventricular events compared to intravenous administration. However, even i.m. injections of saline induced more VPCs compared with intravenous administration of cells, suggesting a pro-arrhythmic effect of the direct injection. During the acute phase, i.m. injections could act as a trigger for VPCs and VT irrespective of the presence or absence of cells in the saline solution. The mechanism seems to be related to mechanical injury inflicted by needle, as shown by the presence of inflammatory cells and white foci. To better evaluate the electrophysiological changes, we analyzed QT time and QTc time with respect to different groups of treatment. During injections, the QT and QTc times were prolonged in all 3 groups. However, at Day 7, the QTc was prolonged only in the two groups that were treated with i.m. injections. During ischemia, prolongation of QT and QTc time was strongly related to the ischemia/reperfusion injury. Nevertheless, at Day 7, the QTc time was prolonged only in the 2 groups treated with i.m. injection, independently of the presence or absence of cells. The prolonged QTc times registered in the group of animals treated with i.m. injections suggested that the injections were associated with changes in cardiac electrophysiological properties. It is well known that ischemia induces a prolongation of QT interval due to prolonged action potential duration, ${ }^{14,15}$ and that QT prolongation and QT variation are strongly related to sudden cardiac death. ${ }^{16,17}$ Heterogeneity in conductance time and recovery period represents a dangerous background for re-entry arrhythmias. In our population, the QTc prolongation was induced by electrical remodeling probably related to the inflammatory substrate induced by needle injury. This hypothesis needs to be confirmed by further studies evaluating serial histological changes after cell injections.

Data recorded at Day 21 from ischemia induction showed a greater number of VPCs and a persisting QTc time prolongation in animals treated with i.m. injections of BMSCs compared with animals treated with i.m. saline, suggesting a pro-arrhythmic effect strictly related to BMSCs. The pro-arrhythmic status could be related to an incomplete differentiation of cells toward cardiomyocytes. We found isolated groups of cells within the ischemic areas. These islets could promote a mechanical reentry. The differentiation of BMSCs into cardiomyocytes is not always associated with cell-cell communication, which in the myocardium is mediated by many different factors, i.e. gap-junction mediated cell contacts, cell-matrix interactions, and signaling through adhesion molecules..$^{14,15}$ It is plausible that the initial differentiation of cells lacks cell-cell communication. The relative immaturity of cells used for cell transplantation may increase the risk of potential mismatch with the adult host cardiomyocytes. The mechanism of pro-arrhythmic status was related to a mechanical re-entry, due to isolated transplanted cells acting synergistically with the electrical remodeling suggested by the persistence of QT prolongation.

\section{Conclusions}

I.m. injections of BMSCs induced an electrical instability, as shown by a high number of VPCs and a longer QTc time, as compared with i.m. injections of saline and with intravenous administration of BMSCs. The pro-arrhythmic status was related to the needle injury during injections and to the electrical remodeling induced by BMSCs administration. These preliminary data need to be confirmed by further studies evaluating a longer follow up before translating information into clinical studies.

\section{References}

1. Schächinger V, Erbs S, Elsässer A, et al. Intracoronary bone marrow-derived progenitor cells in acute myocardial infarction. N Engl J Med 2006;355:1210-21.

2. Tendera M, Wojakowski W, Ruzyłło W, et al. Intracoronary infusion of bone marrowderived selected CD34+CXCR4+ cells and non-selected mononuclear cells in patients with acute STEMI and reduced left ventricular ejection fraction: results of randomized, multicentre Myocardial Regeneration by Intracoronary Infusion of Selected Population of Stem Cells in Acute Myocardial Infarction (REGENT) Trial. Eur Heart J 2009;30:1313-21.

3. Strauer BE, Steinhoff G. 10 years of intracoronary and intramyocardial bone marrow stem cell therapy of the heart: from the methodological origin to clinical practice. J Am Coll Cardiol 2011;58:1095-104.

4. Strauer BE, Yousef M, Schannwell CM. The acute and long-term effects of intracoronary Stem cell Transplantation in 191 patients with chronic heARt failure: the STAR-heart study. Eur J Heart Fail 2010;12: 721-9.

5. Quyyumi AA, Waller EK, Murrow J. CD34(+) cell infusion after ST elevation myocardial infarction is associated with improved perfusion and is dose dependent. Am Heart J 2011;161:98-105.

6. Menaschè $P$. Stem cell therapy for heart failure: are arrhythmias a real safety concern? Circulation 2009;119:2735-40.

7. Kim SH, Pak HN, Park JH, et al. Cardiac cell therapy with mesenchymal stem cell induces cardiac nerve sprouting, angiogenesis, and reduced connexin43-positive gap junctions, but concomitant electrical pacing increases connexin43-positive gap junctions in canine heart. Cardiol Young 2010;20:30-317.

8. Menasché $\mathrm{P}$, Alfieri 0 , Janssens $\mathrm{S}$, et al. The Myoblast Autologous Grafting in Ischemic Cardiomyopathy (MAGIC) Trial. First randomized placebo-controlled study of myoblast transplantation. Circulation 2008;117:1189-200.

9. Brunskill SJ, Hyde CJ, Doree CJ, et al. Route of delivery and baseline left ventricular ejection fraction, key factors of bonemarrow-derived cell therapy for ischaemic heart disease. Eur J Heart Fail 2009;11: 887-96.

10. Makela J, Antills V, Ylitalo K, et al. Acute 
homing of bone marrow-derived mononuclear cells in intramyocardial vs. intracoronary transplantation. Scand Cardiovasc J 2009;43:366-73.

11. Abdel-Latif A, Bolli R, Tleyjeh IM. Adult bone marrow-derived cells for cardiac repair: a systematic review and metaanalysis. Arch Intern Med 2007;167:98997.

12. Menasche P. Cardiac cell therapy: lessons from clinical trials. J Mol Cell Cardiol

\section{1;50:258-65.}

13. Sagie A, Larson MG, Goldberg RJ, et al. An improved method for adjusting the QT interval for heart rate (the Framingham Heart Study). Am J Cardiol 1992;70:797801.

14. Tirziu D, Giordano FJ, Simons M. Cell communications in the heart. Circulation 2010;122:928-37.

15. Redwood SR, Taggart PI, Sutton PM, et al. Effect of magnesium on the monophasic action potential during early ischemia in the in vivo human heart. J Am Coll Cardiol 1996;28:1765-9.

16. De Bruyne MC, Hoes AW, Kors JA, et al. QTc dispersion predicts cardiac mortality in the elderly: the Rotterdam Study. Circulation 1998;97:467-72.

17. He JQ, Ma Y, Lee Y, et al. Human embryonic stem cells develop into multiple types of cardiac myocytes action potential characterization. Circ Res 2003;93:32-9. 